\title{
Willingness and Obstacles of Food and Farming Education in Leisure Farm Management: Viewpoint of Experience Activities
}

\author{
Wan-Chi Yang1, Chun-Nan Lin², Pi-Hsia Hu${ }^{3 *}$ \\ ${ }^{1}$ Department of Hospitality Management, MingDao University, Taiwan \\ ${ }^{2}$ Department of Agribusiness Management, National Pingtung University of Science and Technology, Taiwan \\ ${ }^{3}$ Department of Tropical Agriculture and International Cooperation, National Pingtung University of Science and Technology, \\ Taiwan \\ Email: ^bihshya_hwu@hotmail.com
}

How to cite this paper: Yang, W.-C., Lin, C.-N. and Hu, P.-H. (2020) Willingness and Obstacles of Food and Farming Education in Leisure Farm Management: Viewpoint of Experience Activities. Open Access Library Journal, 7: e6485.

https://doi.org/10.4236/oalib.1106485

Received: May 29, 2020

Accepted: June 27, 2020

Published: June 30, 2020

Copyright $\odot 2020$ by author(s) and Open Access Library Inc.

This work is licensed under the Creative Commons Attribution International License (CC BY 4.0).

http://creativecommons.org/licenses/by/4.0/

\begin{abstract}
In recent years, issues related to food and farming education have been gradually paid attention to. From the perspective of leisure farm operators, this study analyzes the willingness and obstacles of leisure farms to promote food and farming education and provides new options for the agricultural government to implement food and farming education. The results show that operators' willingness to operate food and farming education includes factors. Such as farmland size, years of establishment, number of employees, and operating income; obstacles include external government regulations, internal factors such as environment, human resources, and talent. Obstacles will affect the operator's willingness to operate education for food farmers. Therefore, leisure farms can increase human resources, strengthen professional explanations, and develop experiential teaching models, becoming the best way to promote food education.
\end{abstract}

\section{Subject Areas}

Business \& Economics

\section{Keywords}

Leisure Farms, Food and Farming Education, Willingness and Obstacles, Experience Activities

\section{Introduction}

Since 2006, food prices have continued to rise, extending problems such as riots, 
famine, and malnourished population growth; on the other hand, according to the 2014 report of the United Nations Climate Change Commission (IPCC), climate change is also impacting food, and human security is a big crisis for Taiwan that depends on imports of food. According to the information of the Agricultural Committee of the Executive Yuan, Taiwan's food self-sufficiency rate in 2017 was $32.2 \%$ [1]. If Taiwan continues to rely on imports, failure to find a solution will have a significant impact on Taiwan's economic development and social stability.

Agriculture is the foundation of region's industry, but Taiwan's agriculture is facing severe challenges of arable land loss, aging rural labor force, and climatic and environmental changes. Therefore, the industry, government, and academia are all looking for solutions, including excellent agriculture, technology agriculture, and leisure agriculture. Taking leisure agriculture as an example, consumer contact can give consumers more opportunities to learn about the characteristics and advantages of local agricultural products. And then use and consume local agricultural products, which is an essential opportunity for the sustainable development of Taiwan's agriculture [2]. The Department of Leisure Agriculture combines natural landscape and ecological resources in the agricultural environment, agricultural production (process), agricultural and fishery products, rural culture, humanities and arts, rural facilities, tourism, and other resources to develop new services with leisure functions industry. This means that the "Leisure" products or services it provides must be based on "Agriculture".

In recent years, the issues related to "food farmers", "food security" and "food education" have been valued by the people. The sources and production methods of food have become the main concerns of the people. The industrialized food production and sales methods have caused many problems, including human health and the ecological environment. The problem of "food safety" has gradually emerged, making developed countries realize the importance of food security. The national social awareness of agriculture has gradually reversed. The requirements for the source control of agricultural products and dietary safety have also increased [3]. The concept has taken root and spread to schools and families, teaching the importance of traceability of ingredients. Leisure agriculture should be able to provide fresh, hygienic, and safe agricultural products to meet the needs of repairers. Conserve natural ecological landscapes, conserve water sources, regulate the microclimate, and improve the environment in which people live and provide another need for urban social and cultural life. The three functions of production, ecology, and life, such as leisure agriculture, farming culture, and folk culture tourism, can provide consumers with agricultural knowledge education and demonstration functions.

Tuan [4] once put forward the viewpoint of the economics of experience of leisure agriculture, thinking that to experience the fun of doing it yourself, "experience" and "feel" become commercial products that can be sold. Consumers' internal psychological feelings come from personal The interaction of mood and 
events, and through the design and operation of agricultural experience activities, can increase the sales opportunities of individual farm products. Leisure agriculture is based on life and situation, shaping the sensory experience and thinking identification, and finding new survival value and space for commodities. Allow visitors to experience special agricultural production activities, such as picking vegetables and fruits, making tea, feeding, fishing, agricultural products processing, etc.; the industry persuaded consumers to pay for the experience with carefully designed situations. At the same time, more and more consumers are willing to spend money to buy the experience. However, after two or thirty years of development, the design of experiential activities has gradually entered into rigid imprisonment. There is an urgent need for innovative thinking and methods to make new attempts for experiential activities. The new design method of experiential activities has become the new educational significance of leisure agriculture.

Therefore, this study will explore the new direction of leisure farm experience activity design from the perspective of leisure farm operators, analyze the willingness and obstacles of leisure farms to implement food education, and provide new options for agricultural administration to implement food education.

\section{Literature Review}

\subsection{Food and Farming Education}

Shih [3] said that the term "food education" was a newly coined word proposed by the Japanese as early as 1896 and 1898 . The work mentioned, "sports, intellectual education, and talent education are food education". Yang [5] pointed out that in 1903, the Japanese village Jingxianzhai pointed out in the serial novel "Esophagus" that food education is the first among children's moral education, intellectual education, sports, and food education. Food consumption is inseparable from agricultural production and sales. Agriculture is the most crucial source of human food, and dietary activities are a part of agricultural activities. "Food and agriculture" are essentially one and two sides. Therefore, the term "food and farming education" was born [6].

Chen [7] mentioned that the form of food and farming education comes from Japan's six-level industrial policy. In order to improve the development of the rural economy, Japan implements a six-level industrial policy to strengthen the integration of agriculture, forestry and fishery production, processing and sales, and enhance the added value of agriculture; to revitalize the agricultural, fishing, and mountain villages and promote the local consumption of real estate. The demand for nutritious lunches in high schools, middle schools, and primary schools promotes the consumption of agricultural products in rural areas. A special law is established to require school nutritious lunches to use local ingredients and set goals; through dietary education, to enhance their public confidence in consuming domestic agricultural products, let the citizens feel the local 
natural food culture, build a cooperative model and functional relationship between producers, direct sales offices and schools, and stimulate students' emotions about producers and food supply; to save transportation costs and contribute to the environment.

Lin [2] said that "food and farming education" is a broadly defined name. The concept of this social movement is established. For example, government agencies, schools, peasant associations, non-profit organizations, leisure farms, organic farms, and agricultural enterprises can pass through Cooperate and participate in typical development. Moreover, integrate the elements of education into business behavior, combine the concept of agricultural experience and health, promote the way of connecting with the local agricultural food network, and cooperate with environmentally friendly and organic production methods [6]. It should be the future of agricultural product marketing - the direction of development.

\subsection{Leisure Farm}

The original meaning of leisure farms [8], that is, the "Leisure" products or services it provides, must be based on "Agriculture" operation. Therefore, the development of leisure farms is different from general sightseeing and tourism type. In addition to maintaining the production function of traditional agriculture, it is also necessary to transform some agricultural resources into leisure and recreation functions based on local characteristics.

Many researchers have two different perspectives on leisure farms. First, from the perspective of agricultural management, scholar Chiang [9] emphasized that leisure agriculture is a type of agricultural management transformed from production or combined with the service industry. Another scholar, Lin, Chen and Wang [10], looked at the service industry as a leisure service industry. He believed that leisure farms consisted of farmers engaged in agricultural production activities and living in rural areas. The site, following the local terrain, climate, soil, hydrology, and other natural environments, through appropriate planning and design, engaged in the local leisure service business, and then play the local leisure function. The primary source of profit for leisure farms is not the production and sales of agricultural products or the sale of processed products, but the income from operating the service industry, which has exceeded the scope of traditional agricultural production.

\subsection{Willingness and Obstacles}

Woodside and Lysonski [11] pointed out that the formation of will is caused by the interaction of many complex factors, whether social, economic, or psychological factors affect a person's will. Ajzen [12] emphasizes that willingness is the most direct factor in determining behavior and the best measurement tool for predicting behavior. Therefore, in order to discuss the willingness of leisure farm operators to operate food agriculture education, such as the benefits of food 
agriculture education on-farm operation, sustainable agriculture, and circular economy, the values of Archibald [13] and Woodside and Lysonski [11], the emotional component of Meng [1] and the action intention of Bartram and Joshua [10] are used as the measurement facets.

Zheng Weizheng [14] pointed out that business obstacles may be affected by various internal, external, and other factors. It causes management problems and factors that lead to their unwillingness to operate, including legal policies, market participation, operating costs, investment funds, social responsibility, human resources, field facilities, event travel planning, professional commentators, etc.

\subsection{Experience Activities}

In the era of the experience economy, only providing products or services can no longer meet the needs of consumers [15]. What consumers demand is a personalized experience outside of products and services. Everyone's experience and background are different, and their feelings are also different. The designed experience activities should have market segmentation and differentiation, give consumers intimate products and services, and create unforgettable and pleasant experiences.

Tuan [4] pointed out that the experience economy emphasizes that the value of products or services is to provide customers with the opportunity to experience and create a better feeling; experience activities must be able to touch the customer's senses (eyes, ears, nose, tongue, skin), and there are five senses of sight, hearing, smell, taste, touch, etc. The customer's experience comes from personal participation, so the product or service should retain the customer's personal experience. Because each customer's characteristics and life experiences are different, it is necessary to tailor-made experience activities for customers, and products and services should be diversified; industries in rural areas are mainly based on agricultural and agricultural resources. Because agricultural resources are biological and rural resources are living, there is a clear difference between industries in rural areas and industries in urban areas.

Implementing experience can connect abstract knowledge to life ability, and even internalize experience into beliefs and actions. Through experiential education, food farmers' education attempts to promote the awareness and attention of the people and the next generation on this issue. Tung [16] pointed out: "The education of food farmers is a process of experiential education. Learners learn about local agriculture, the correct way of life, and their diet through an interactive experience with food, farmers, rural areas, and related actors." The culture formed, and the impact of agriculture and diet on the ecological environment. "Therefore, the education of food farmers is an education that emphasizes experience". Educational philosopher Dewey once said: "education is life, life is education." Reflecting on the experience of actual participation can allow people to build knowledge, enhance skills, and self-worth from this, and this concept coincides with the goal of food and farming education. 


\section{Research Design}

\subsection{Research Framework and Assumptions}

According to the research literature, the established research framework is discussed. The adopted structure includes the characteristics of leisure farms, the willingness to operate food and farming education, and the obstacles to the management of food and farming education; [4] can be divided into farm areas according to the characteristics of leisure farms. The five items of farm location, the establishment of time, land scale, annual operating income, and number of employees; the business willingness of food and farming education includes three items of value, emotion, and action intention; the operational obstacles to food and farming education affect leisure farms There are two external and internal projects, as shown in the architecture diagram (Figure 1).

\subsection{Questionnaire Design and Sampling}

The questionnaire's content is divided into three parts, which are five questions about the characteristics of leisure farms, including the farm's location, the scale of the farm, the number of years of establishment of the farm, the number of employees, and the annual operating income. Sixteen questions about the willingness of the industry to operate food and farming education, including values, emotional components, and action intentions, are three significant aspects-thirteen obstacle factors, including external factors and internal factors. Then the questionnaire's content, according to the reference scholars' revisions of the characteristics of leisure farms by scholar Tuan [4], the business willingness by scholar Archibard [13], scholar Bartram and Joshua [17], scholar Woodside and Lysonski [11], and the operational obstacles by scholar Tuan [4], scholar Zheng [14], and based on the opinions of experts and scholars revised. Use Likert's five-point scale.

Based on the survey of the Taiwan Leisure Agriculture Society in 2007, the number of 1244 farms registered as leisure farms was used as the matrix. Based on the research time and research funding constraints, the convenience sampling principle is adopted.

According to the confidence level is $95 \%$, and the margin of error is 0.05 . to measure a sample of consumer population in this research using Slovin formula [18].

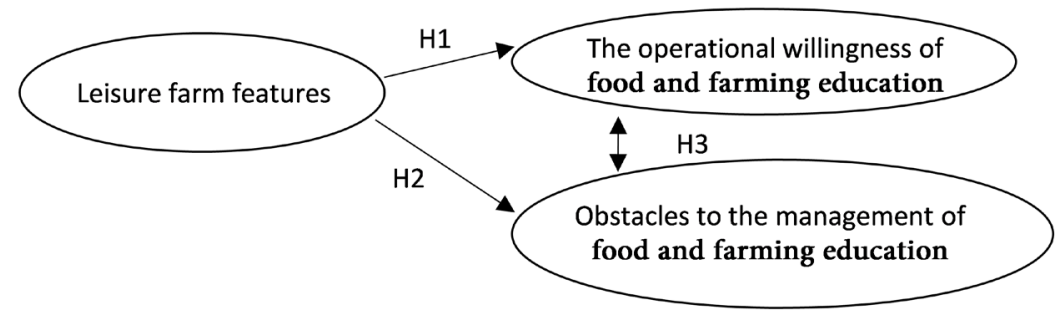

Figure 1. Research architecture diagram. Source: This study. 
The formula of sampling in this method:

$$
n=N /\left(1+\left(N \times e^{2}\right)\right)
$$

where:

$n$ : number of samples

$N$ : number of populations

e: estimated deviation of 0.05

Overall, 303 samples were chosen and usable questionnaires were collected and used for data analysis. Therefore, questionnaires were sent out by mail reply. A total of 313 questionnaires were sent out, and a total of 214 valid questionnaires were returned.

\subsection{Hypothesis and Verification}

The research hypotheses of this study are described as follows:

Hypothesis 1: The characteristics of leisure farms have no significant differences in the willingness to operate food and farming education.

Hypothesis 2: The characteristics of leisure farms have no significant difference in the obstacles to the management of food and farming education.

Hypothesis 3: The willingness of leisure farms to operate food and farming education has no significant correlation with obstacles.

One-way ANOVA is suitable for testing whether there is a difference in average between multiple sets of independent samples, assuming that the independent variable is categorical. The dependent variable must be continuous. Mother, the population must be a normal distribution (Normal Distribution); the number of variations of the two groups of samples must be equal; the samples must be independent variables, and so on.

Variance analysis is a hypothesis test. The null hypothesis is that there is no difference in the average population of each group of samples. The opposite hypothesis is that the average population of at least one group of samples is different from the others.

$$
\begin{aligned}
& \mathrm{H} 0: \mu_{1}=\mu_{2}=\mu_{3}=\cdots=\mu_{k} \\
& \mathrm{H} 1: \quad \mu_{i} \neq \mu_{j}, i, j=1, \cdots, k \\
& \mathrm{SST}=\mathrm{SSR}+\mathrm{SSE} \\
& \mathrm{SSR}=\sum n_{i}\left(\hat{\mu}_{t}-\bar{y}\right)^{2} \\
& \mathrm{SSE}=\sum\left(y_{i}-\hat{\mu}_{t}\right)^{2} \\
& \mathrm{SST}=\sum\left(y_{i}-\bar{y}\right)^{2}
\end{aligned}
$$

Among them, SSR is the variation between groups; SSE is the variation within the group; SST is the total variation; $n_{i}$ is the number of samples in each group; $\mu_{i}$ is the average of each group; $\hat{y}$ is the average of all samples.

Pearson's correlation coefficient (Pearson's correlation coefficient) is a statistical indicator that reflects the close relationship between two sets of variables 
and is used to describe the trend of two sets of linear data changing and moving together. The premise assumes that the two sets of variable data are typically distributed or close to the normal distribution, assuming that there are two variables $\left(x_{i}, y_{i}\right), i=1, \cdots, n$, the formula is as follows

$$
\rho=\frac{\sum_{i=1}^{n}\left(x_{i}-\mu_{x}\right)\left(y_{i}-\mu_{y}\right)}{\sqrt{\sum_{i=1}^{n}\left(x_{i}-\mu_{x}\right)^{2} \sum_{i=1}^{n}\left(y_{i}-\mu_{y}\right)^{2}}}
$$

Correlation coefficient $\rho=-1 \leq p \leq 1, \mu_{x}$ and $\mu_{y}$ represents the average of the variables $x$ and $y$. Their values are between 1 and -1 when the linear relationship between the two variables is enhanced. The correlation coefficient tends to 1 or -1 if the coefficient is greater than 0 . It means a positive correlation. If the coefficient is less than 0 , it means a negative correlation; if the correlation coefficient is equal to 0 , it means there is no linear correlation between them.

\section{Empirical Analysis}

The Cronbach's $\alpha$ values of each facet of this research questionnaire are between 0.763 and 0.892 , indicating that the facets of this scale have excellent reliability. The factor load of business willingness Factor load of 16 variables of business willingness is between 0.483 and 0.875 , and the cumulative total solution variation is $68.737 \%$. This means that each variable can be classified into factors such as value, emotional components, and intention to act. And the Cronbach's $\alpha$ values of business willingness factor including values is 0.892 , emotional components is 0.804 , and action intentions is 0.802 , indicating that the facets of this scale have excellent reliability. Moreover, it is relevant; the factor load of operating obstacles is between 0.43 and 0.801 , and the cumulative total solution variation is $68.737 \%$, which means that each variable can be classified into external and internal factors, and is related. And the Cronbach's $\alpha$ values of operating obstacles factor including external factors is 0.763 , and internal factors is 0.856 , indicating that the facets of this scale have excellent reliability. The facets of willingness and obstacles are credible and valid.

\subsection{Characteristic Analysis of Leisure Farm}

In this research survey, there is a maximum of 107 farms in the "Northern Region", accounting for $50.0 \%$; the largest farm size is "more than 0.5 hectares-less than 1.0 hectare", a total of 107 , accounting for $50.0 \%$; The largest number of " 6 - 10 years" is 69 , accounting for $32.2 \%$; the number of farm employees is " 3 - 6", which is 120 , accounting for $56.1 \%$; the annual operating income is "less than $1,000,000$ yuan", which is 76 , accounting for $35.5 \%$.

\subsection{Analysis of Factors of Food and Farming Education Management Willingness}

According to the verification results of single-factor variance analysis (as shown in Table 1): the leisure farm operators at different farm locations have no 
Table 1. Comparison of differences in characteristics of leisure farms to factors of willingness to operate.

\begin{tabular}{|c|c|c|c|c|}
\hline Leisure farm features & Measure facets & F value & P-value & $\begin{array}{c}\text { Scheffe } \\
\text { verification }\end{array}$ \\
\hline Farm location & Values & 0.145 & 0.933 & \\
\hline 1. Northern Region & $\begin{array}{l}\text { Emotional } \\
\text { component }\end{array}$ & 2.258 & 0.083 & \\
\hline 2. Central Region & $\begin{array}{l}\text { Behavior } \\
\text { intention }\end{array}$ & 2.068 & 0.106 & \\
\hline \multicolumn{5}{|l|}{ 3. Southern Region } \\
\hline \multicolumn{5}{|l|}{ 4. Eastern Region } \\
\hline Farmland scale & Values & 3.062 & $0.029^{*}$ & \\
\hline 1. Above 0 hectares-less than 1.0 hectares & $\begin{array}{l}\text { Emotional } \\
\text { component }\end{array}$ & 2.051 & 0.108 & \\
\hline 2. Above 1.0 hectares-less than 10 hectares & $\begin{array}{l}\text { Behavior } \\
\text { intention }\end{array}$ & 4.513 & $0.004^{* *}$ & $2>1$ \\
\hline \multicolumn{5}{|l|}{ 3. Above 10 hectares-less than 50 hectares } \\
\hline \multicolumn{5}{|l|}{ 4. 50 hectares or more } \\
\hline Year of the establishment of the farm & Values & 7.219 & $0.000^{* *}$ & $2,3,4>1$ \\
\hline 1. Under 5 years & $\begin{array}{l}\text { Emotional } \\
\text { component }\end{array}$ & 12.983 & $0.000^{* *}$ & $2,3,4,5>1$ \\
\hline 2. 6 - 10 years & & & & $4>3$ \\
\hline 3. $11-15$ years & $\begin{array}{l}\text { Behavior } \\
\text { intention }\end{array}$ & 9.320 & $0.000^{* *}$ & $2,3>1$ \\
\hline \multicolumn{5}{|l|}{ 4. 16 - 20 years } \\
\hline \multicolumn{5}{|l|}{ 5. 21 years or more } \\
\hline Number of farm employees & Values & 1.712 & 0.148 & \\
\hline 1. 2 people or less & $\begin{array}{l}\text { Emotional } \\
\text { component }\end{array}$ & 1.635 & 0.167 & \\
\hline 2.3 - 6 people & $\begin{array}{l}\text { Behavior } \\
\text { intention }\end{array}$ & 7.319 & $0.000^{* *}$ & $4>1$ \\
\hline 3. 7 - 10 people & & & & $4>2$ \\
\hline \multicolumn{5}{|l|}{ 4. 11 - 14 people } \\
\hline \multicolumn{5}{|l|}{ 5. 15 people or more } \\
\hline Farm operating income & Values & 6.257 & $0.000^{* *}$ & $2,4>1$ \\
\hline 1. Less than $1,000,000 \mathrm{NT}$ & $\begin{array}{l}\text { Emotional } \\
\text { component }\end{array}$ & 3.721 & $0.003^{* *}$ & $2>3$ \\
\hline 2. $1,000,000 \mathrm{NT}-5,000,000 \mathrm{NT}$ & $\begin{array}{l}\text { Behavior } \\
\text { intention }\end{array}$ & 8.627 & $0.000^{* *}$ & $2,4,5>1$ \\
\hline \multicolumn{5}{|l|}{ 3. $5,000,000 \mathrm{NT}-10,000,000 \mathrm{NT}$} \\
\hline \multicolumn{5}{|l|}{ 4. $10,000,000 \mathrm{NT}-15,000,000 \mathrm{NT}$} \\
\hline \multicolumn{5}{|l|}{ 5. $15,000,000 \mathrm{NT}-20,000,000 \mathrm{NT}$} \\
\hline 6. $20,000,000 \mathrm{NT}$ or more & & & & \\
\hline
\end{tabular}

Note: ${ }^{\star}$ means $\mathrm{P}<0.05 ;{ }^{* *}$ means $\mathrm{P}<0.01$; Source: This study. 
different willingness to operate food and farming education. In the field of farmland, the "value concept" ( $\mathrm{F}=3.062, \mathrm{p}<0.05)$ and the "behavior intention" $(\mathrm{F}=4.513, \mathrm{p}<0.01)$ are both significantly different. According to Scheffé's hindsight, the farmland regulations Recreation farm operators in "1.0 hectares-less than 10 hectares" are more likely to agree with "intention to behavior" than those in "0.5 hectares-less than 1.0 hectare" In the years of establishment of the farm, "value concept" ( $F=7.219, \mathrm{p}<0.01)$, "emotional component" ( $\mathrm{F}=$ $12.983, \mathrm{p}<0.01)$ and "behavior intention" $(\mathrm{F}=9.320, \mathrm{p}<0.01)$. All have significant differences. After Scheffé learned afterward, there are significant differences between "value concept", "emotional component" and "behavior intention", showing that those who have been established for more than six years compared with those established for less than five years Business owners have a higher willingness to operate food and agriculture education. In terms of the number of employees on the farm, there was a significant difference in "behavior intention" $(\mathrm{F}=7.319, \mathrm{p}<0.01)$. According to Scheffé, it was later learned that leisure farm operators with 11 - 14 farm employees were more than leisure farms with less than six employees. Practitioners' "intention to conduct" is more agreeable. In the annual operating income component, "value concept" $(\mathrm{F}=6.257, \mathrm{p}<0.01)$, "emotional component" ( $\mathrm{F}=3.721, \mathrm{p}<0.01)$ and "behavior intention" $(\mathrm{F}=$ 8.627, $\mathrm{p}<0.01)$ There is a significant difference between them. Scheffé later learned that there was a significant difference between "value concept", "emotional component" and "behavior intention", showing that the higher the annual operating income of the leisure farm industry, the annual operating income is less than 1,000,000 Yuan's leisure farm operators have a higher willingness to operate food and agriculture education.

\subsection{Analysis of Factors of Food and Farming Education Management Obstacles}

According to the verification results of the single-factor analysis of variance (as shown in Table 2), the operation obstacles for leisure farm operators in different farm locations to operate food education are different because the farms are located in different regions. In terms of farmland size, the "external factors" ( $F=$ 5.836, $\mathrm{p}<0.01$ ) are significantly different. After Scheffé's hindsight, it is known that the farmland size is "more than 0.5 hectares-less than 1.0 hectare" for leisure farm operators. The "external factors" that hinder the operation of leisure farm operators compared to "1.0 hectares-less than 10 hectares" are more obvious. In the years of establishment of the farm, the "external factors" ( $F=22.176, p<$ $0.01)$ and the "internal factors" ( $F=18.097, \mathrm{p}<0.01)$ are both significantly different. After the Scheffé comparison, we learned that there are significant differences between "external factors" and "internal factors", indicating that the obstacles to the education and management of food farmers are due to the years of farm establishment. In terms of the number of employees on the farm, the "intrinsic factor" ( $\mathrm{F}=8.879, \mathrm{p}<0.01)$ is significantly different. According to 
Table 2. Comparison of differences in characteristics of leisure farms against obstacles.

\begin{tabular}{|c|c|c|c|c|}
\hline Leisure farm features & Measure facets & F value & P-value & $\begin{array}{c}\text { Scheffe } \\
\text { verification }\end{array}$ \\
\hline Farm location & External factor & 2.144 & 0.096 & \\
\hline 1. Northern Region & Internal factors & 2.433 & 0.066 & \\
\hline \multicolumn{5}{|l|}{ 2. Central Region } \\
\hline \multicolumn{5}{|l|}{ 3. Southern Region } \\
\hline \multicolumn{5}{|l|}{ 4. Eastern Region } \\
\hline Farmland scale & External factor & 5.836 & $0.001^{* *}$ & $1>2$ \\
\hline 1. Above 0 hectares-less than 1.0 hectares & Internal factors & 1.498 & 0.216 & \\
\hline \multicolumn{5}{|l|}{ 2. Above 1.0 hectares-less than 10 hectares } \\
\hline \multicolumn{5}{|l|}{ 3. Above 10 hectares-less than 50 hectares } \\
\hline \multicolumn{5}{|l|}{ 4. 50 hectares or more } \\
\hline Year of the establishment of the farm & External factor & 22.176 & $0.000^{* *}$ & $2,3,4,5>1$ \\
\hline 1. Under 5 years & & & & $4>2,3$ \\
\hline $2.6-10$ years & & & & $5>1,3$ \\
\hline 3. $11-15$ years & Internal factors & 18.097 & $0.000^{* *}$ & $2>1,3$ \\
\hline 4. 16 - 20 years & & & & $4>2,5$ \\
\hline 5. 21 years or more & & & & $5>4$ \\
\hline Number of farm employees & External factor & 1.195 & 0.314 & \\
\hline 1. 2 people or less & Internal factors & 8.879 & $0.000^{* *}$ & $4>1,2$ \\
\hline \multicolumn{5}{|l|}{ 2. 3 - 6 people } \\
\hline \multicolumn{5}{|l|}{ 3. 7 - 10 people } \\
\hline \multicolumn{5}{|l|}{ 4. 11 - 14 people } \\
\hline \multicolumn{5}{|l|}{ 5. 15 people or more } \\
\hline Farm operating income & External factor & 6.135 & $0.000^{* *}$ & $1,2>3$ \\
\hline 1. Less than $1,000,000 \mathrm{NT}$ & Internal factors & 6.934 & $0.000^{* *}$ & $5>1,2$ \\
\hline \multicolumn{5}{|l|}{ 2. $1,000,000 \mathrm{NT}-5,000,000 \mathrm{NT}$} \\
\hline \multicolumn{5}{|l|}{ 3. $5,000,000 \mathrm{NT}-10,000,000 \mathrm{NT}$} \\
\hline \multicolumn{5}{|l|}{ 4. $10,000,000 \mathrm{NT}-15,000,000 \mathrm{NT}$} \\
\hline \multicolumn{5}{|l|}{ 5. $15,000,000 \mathrm{NT}-20,000,000 \mathrm{NT}$} \\
\hline $6.20,000,000$ or more & & & & \\
\hline
\end{tabular}

Note: ${ }^{\star}$ means $\mathrm{P}<0.05 ;{ }^{* *}$ means $\mathrm{P}<0.01$; Source: This study.

Scheffé, it is later learned that leisure farm operators with 11 - 14 employees on the farm are less than leisure farms with less than six employees. The "intrinsic factors" of business obstruction of the operators are different. In the annual operating income part, the "external factors" $(\mathrm{F}=6.135, \mathrm{p}<0.01)$ and the "internal factors" ( $\mathrm{F}=6.934, \mathrm{p}<0.01)$ are significantly different. After Scheffé's hindsight, we learned that there is a significant difference between "external factors" and 
"internal factors", which shows that the obstacles to the education and management of food farmers are affected by the farm's annual operating income.

\subsection{Correlation Analysis of Barriers and Management Willingness of Food Farmer Education Management on Leisure Farms}

Pearson correlation coefficient analysis results (Table 3) show that there is a significant positive correlation between leisure farm operators' management obstacles to food farmers' education and the management willingness facet variables $(\mathrm{r}=0.599, \mathrm{p}<0.01)$. It will affect the willingness of leisure farm operators to operate food education.

\section{Conclusions}

\subsection{Conclusions and Recommendations}

According to the research results, it is found that there are differences in the willingness of different leisure farm operators in the education and management of food farmers. It can be seen that the willingness of food farmers to operate education will be affected by the size of the farmland, the number of years of establishment of the farm, the number of farm employees, and the annual operating income. Food and farming education is an experimental education process that emphasizes "hand-made". Through experience, it teaches people to re-establish the relationship between people, food, and land, to understand the importance of food and agriculture, and to make the right choices for the diet. Therefore, the larger the scale of the land, the longer the farm is established, the more farm employees and the annual operating income, the compound leisure farm operators will have more resources to cooperate in food and farming education. The higher the willingness, there is a clear difference in the behavior image of the food farmers' educational willingness, which shows that the leisure farmer agrees on the management willingness and has the responsibility to do this business after deciding the food farmers' education goals. In other words, the leisure farm operator is an essential part of the promotion of food agriculture education. Leisure farm operators believe that although they have the will to operate food and farming education, they cannot fully develop it. Most business operators operate food and farming education because they have a high connection with the current season's crop, and the relationship between food and agricultural production.

Secondly, leisure farm operators have differences in the obstacles to the education and management of food farmers. It can be seen that the obstacles to the

Table 3. A relevant analysis of leisure farm management obstacles and willingness.

\begin{tabular}{ccc}
\hline & Obstacles & Operating will \\
\hline Obstacles & 1 & \\
Operating will & $0.599^{* *}$ & 1 \\
\hline
\end{tabular}

Note: **means $\mathrm{P}<0.01$; Source: This study. 
education and management of food farmers will be affected by the size of the farmland, the number of years of establishment of the farm, the number of employees on the farm, and the annual operating income. Including external government regulations, the environmental aspects of the internal leisure farm itself, lack workforce, and low level of professional training.

Besides, leisure farm operators have a significant correlation between the willingness of food farmers' education management and the obstacles to management. In other words, business obstacles will affect the willingness of leisure farm operators to operate education for food farmers.

Because of the above, leisure farm operators agree that they will have the responsibility to do this business well after deciding on the food farmers' education goals. Today's Taiwan's eating habits are full of exquisite and refined foods, but they are not necessarily real foods; we have eaten many additives, which may be more tasteful, but in fact, they are the source of many civilization diseases, Is the biggest threat to people's health. Therefore, [19] used food and farming education to have eight characteristics of personal production, local consumption, agricultural food, whole food utilization, family eating, first sensory experience, cultural heritage, and diversity, emphasizing the local production of agricultural products sports. Becoming the taste of real food will be the best spirit to promote food farmers' education.

Recreational farm operators agree that in terms of obstacles, leisure farms should increase the workforce to promote food and farming education, strengthen professional food and farming education interpretation capabilities, and develop a teaching model of experiential learning. Hands-on learning can create learners' motivation for learning. By combining exciting hand-made courses guided by agricultural and environmental issues, the learning effect can be deepened.

\subsection{Limitation of Research}

Based on the research time and research funding constraints, the convenience sampling principle is adopted.

In addition, questionnaires were sent out by mail, leisure farms manager may have a low understanding of food and farming education, which makes it difficult to answers questionnaires.

\section{Conflicts of Interest}

The authors declare no conflicts of interest regarding the publication of this paper.

\section{References}

[1] Meng, Y. (2013) Research on the Influence of Competitive Network on Enterprise's Innovation Willingness. Master Thesis, Harbin Engineering University, Harbin.

[2] Lin, C.N. (2019) Food and Farming Education: Thinking and Patterns of Agricultural Experience Activity Design. Proceedings of the 2019 Cross-Strait Development 
of Leisure Agriculture Conference, Haikou, 17 August 2019, 340-346.

[3] Shih, U.H. (2009) Food Education in Japan. Food and Nutrition in China, 10, 4-7.

[4] Tuan, C.L. (2018) Leisure Agriculture: An Experience Perspective. 3rd Edition, Far Du, Taipei.

[5] Yang, M.C. (2014) Japan's Heavy Food Education High School Students Change Food King. CNA New World, 72, 53-55.

[6] Lai, E.J. (2015) Food and Farming Education in Japan's Central System. Agricultural Extension Anthology, 60, 7-14.

[7] Chen, Y.W. (2012) Japan's Sextiary Industry Policy and its Enlightenment to my Country's Agricultural Policy. Agriculture Policy \& Review, 239, 81-89.

[8] Chen, C.L. (1996) Development of Leisure Agriculture. Nature Magazine, 50, 5-13.

[9] Chiang, R.C. (1989) Operati on and Managemtn of Leisure Farm. Record of Conference of Leisure Agriculture Development. Taipei, 227-236.

[10] Lin, H.P., Chen, C.C. and Wang, J.S. (2007) Study in the Development and Developing Strategy of Taiwan Leisure Farm. Journal of Tpec Press, 15, 152-163.

[11] Woodside, A.G. and Lysonski, S. (1989) A General Model of Traveler Destination Choice. Journal of Travel Research, 17, 8-14. https://doi.org/10.1177/004728758902700402

[12] Ajzen, I. (1985) From Intentions to Actions: A Theory of Planned Behavior. In: Kuhl, J. and Bechkmann, J., Eds., Action Control: From Cognition to Behavior, Springer-Verlag, New York, 11-39. https://doi.org/10.1007/978-3-642-69746-3_2

[13] Archibard, O.H. (1968) On the Concept of Aspiration. Rural Sociology, 33, 484-487.

[14] Zheng, W.Z. (2013) The Main Cause of Private Small and Median Size Enterprises into Operating Predicament. Master Thesis, National Taiwan University, Taipei.

[15] Pine, B.J. and Gilmore, J.H. (1998) Welcome to the Experience Economy. Harvard Business Review, 76, 97-105.

[16] Tung, S.J. and Tsai, C.J. (2014) Planning of Teaching Design of Food and Farming Education for Farmers at the Grassroots Level. Agricultural Extension Anthology, 61, 47-55.

[17] Bartram, F.M. and Joshua, K. (1997) The Folk Concept of Intention. Journal of EXperimental Social Psychology, 33, 101-121. https://doi.org/10.1006/jesp.1996.1314

[18] Slovin, E. (1960) Slovin's Formula for Sampling Technique. https://prudencexd.weebly.com/

[19] Tung, S.J. (2015) The Connection between Japanese Food and Farming Education and Taiwan Agricultural Extension System. Agricultural Extension Anthology, 60, 1-6. 